\title{
SOME REMARKS OF RANDOM GRAPHS
}

\author{
József Túri \\ associate professor, University of Miskolc, Institute of Mathematics \\ 3515 Miskolc, Miskolc-Egyetemváros, e-mail: matturij@uni-miskolc.hu
}

\begin{abstract}
At the beginning of the paper, the known models are briefly presented (The Barabási-Albert model, Watts-Strogatz model, Erdös-Rényi model). In the later part of the paper, some results are presented, which are valid in the Erdös-Rényi model and are also related to the dominant sets of graphs. These results will be considered further in a later paper (Bacsó et al., 2021).
\end{abstract}

Keywords: Erdös-Rényi model, random graphs, dominant sets

\section{Introduction}

Several random graph models exist. Let us first review the main random graph theory models.

Today's intensively researched model is the Barabási-Albert model: The Barabási-Albert model is an algorithm for generating random Scalefree networks using a preferential attachment mechanism. Several natural and human-made systems, including the Internet, the world wide web, citation networks, and some social networks are thought to be approximately scale-free and certainly contain few nodes (called hubs) with unusually high degree as compared to the other nodes of the network. The Barabási-Albert model tries to explain the existence of such nodes in real networks. The algorithm is named for its inventors Albert-László Barabási and Réka Albert.

Preferential attachment means that the more connected a node is, the more likely it is to receive new links. Nodes with a higher degree have a stronger ability to grab links added to the network. Intuitively, the preferential attachment can be understood if we think in terms of social networks connecting people. Here a link from A to B means that person A "knows" or "is acquainted with" person B. Heavily linked nodes represent well-known people with lots of relations. When a newcomer enters the community, they are more likely to become acquainted with one of those more visible people rather than with a relative unknown. The The Barabási-Albert model was proposed by assuming that in the World Wide Web, new pages link preferentially to hubs, i.e., very well-known sites such as Google, rather than to pages that hardly anyone knows. If someone selects a new page to link to by randomly choosing an existing link, the probability of selecting a particular page would be proportional to its degree.

The Barabási-Albert is a scale-free network: the scale-free network is a network whose degree distribution follows a power law, at least asymptotically. That is, the fraction $\mathrm{P}(\mathrm{k})$ of nodes in the network having $\mathrm{k}$ connections to other nodes goes for large values of $\mathrm{k}$ as

$$
\mathrm{P}(\mathrm{k}) \sim \mathrm{k}^{-\gamma},
$$

where $\gamma$ is a parameter whose value is typically in the range $2<\gamma<3$ (wherein the second moment (scale parameter) $\mathrm{k}-\gamma$ is infinite but the first moment is finite), although occasionally it may lie outside these bounds. 


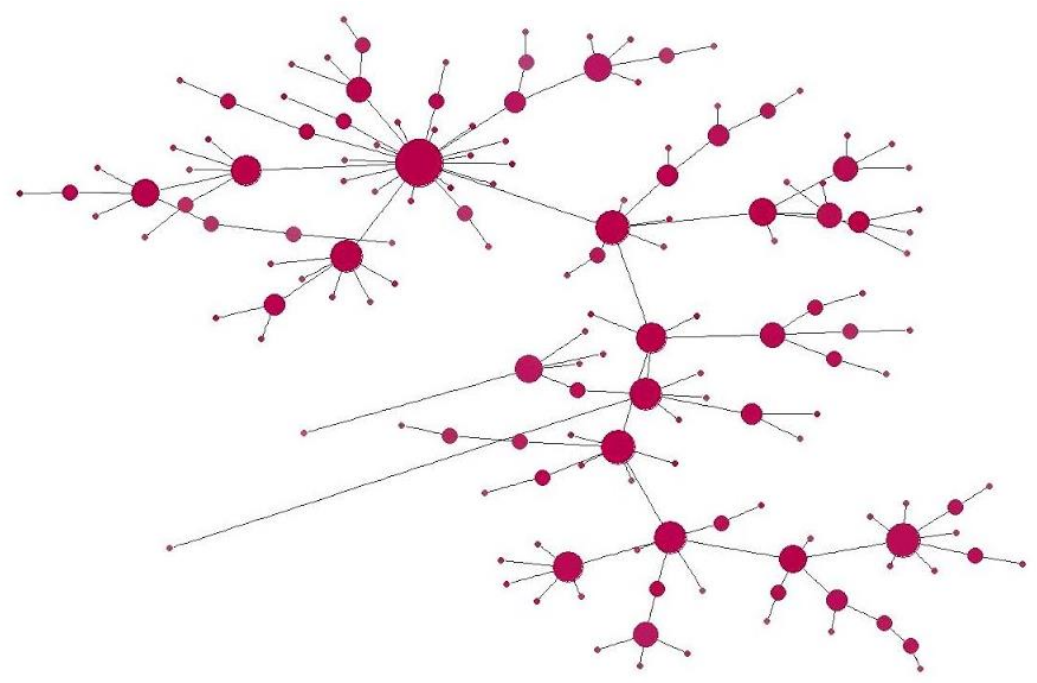

Figure 1. The Barabási-Albert model

The Watts-Strogatz model is a random graph generation model that produces graphs with smallworld properties, including short average path lengths and high clustering. It was proposed by Duncan J. Watts and Steven Strogatz in their article published in 1998 in the Nature scientific journal (Watts and Strogatz, 1998).

Watts-Strogatz model $\mathrm{N}=20, \mathrm{~K}=4, \beta=0.2$

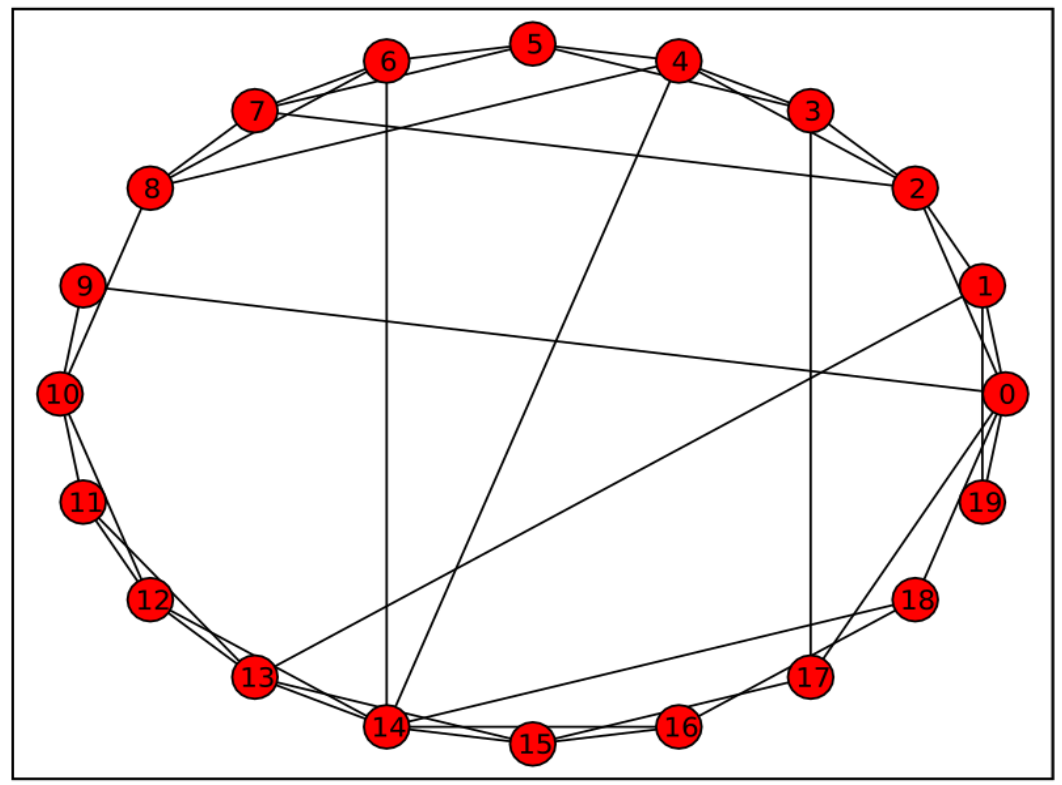

Figure 2. The Watts-Strogatz model

We investigate a classical standard random graph model. This model due to Erdős and Rényi: here every possible edge is created with same p probability: each edge has a fixed probability of being 
present or absent, independently of the other edges. In other work, the probability $\mathrm{p}$ is not constant (Bacsó et al., 2021).

Let us consider the standard random graph model, namely the probability that a pair of vertices (points) is an edge, will be $\mathrm{p}$ and the pairs are completely independent. We take a random graph $\mathrm{G}$ on the vertices $1,2, \ldots, n$.

In the $\mathrm{G}(\mathrm{n} ; \mathrm{p})$ model, a graph is constructed by connecting labelled nodes randomly. Each edge is included in the graph with probability $\mathrm{p}$, independently from every other edge.

With the notation above, a graph in $\mathrm{G}(\mathrm{n} ; \mathrm{p})$ has on average $\left(\begin{array}{l}n \\ 2\end{array}\right)$ p edges.

The distribution of the degree of any particular vertex is binomial:

$$
\mathrm{P}(\operatorname{deg}(\mathrm{v}=\mathrm{k}))=\left(\begin{array}{c}
n-1 \\
k
\end{array}\right) \mathrm{p}^{\mathrm{k}}(1-\mathrm{p})^{\mathrm{n}-\mathrm{k}-1}
$$

where $\mathrm{n}$ is the total number of vertices in the graph.

Since

$$
\mathrm{P}(\operatorname{deg}(\mathrm{v}=\mathrm{k}))=\frac{\left(n p^{3 k_{e}}-n p\right.}{k !},
$$

as $n \rightarrow \infty$ and $n p=$ constant this distribution is Poisson for large $n$.

If $n p>1$, then a graph in $G(n ; p)$ will almost surely have no connected components of size larger than $\mathrm{O}(\log (\mathrm{n}))$.

If $\mathrm{np}=1$, then a graph in $\mathrm{G}(\mathrm{n} ; \mathrm{p})$ will almost surely have largest component whose size order $n^{2 / 3}$.

If $n p \rightarrow c>1$, then a graph in $G(n ; p)$ will almost surely have a unique giant component containing a positive fraction of the vertices. No other component will contain more than $\mathrm{O}(\log (\mathrm{n}))$ vertices.

If $\mathrm{p}<\frac{(1-\varepsilon)^{I n n}}{n}$, then a graph in $\mathrm{G}(\mathrm{n} ; \mathrm{p})$ will almost surely contain isolated vertices and thus be disconnected.

If $\mathrm{p}>\frac{(1-\varepsilon) \ln n}{n}$, then a graph in $\mathrm{G}(\mathrm{n} ; \mathrm{p})$ will almost surely be connected.

$$
\underline{\ln (n)}
$$

This $\frac{\frac{h}{n}}{n}$ is a sharp threshold function for the connectedness of $\mathrm{G}(\mathrm{n} ; \mathrm{p})$.

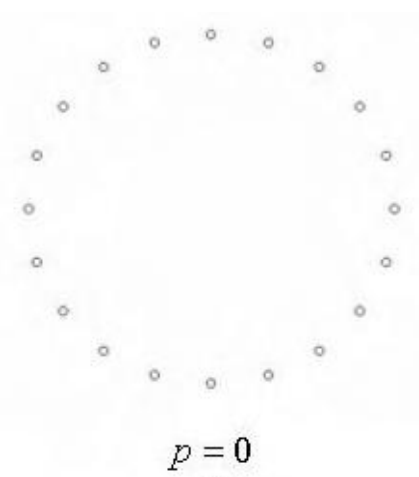

(a)

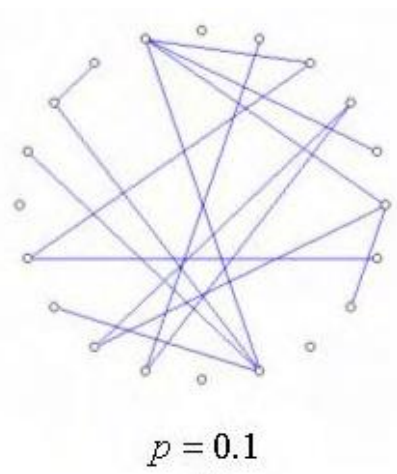

(b)

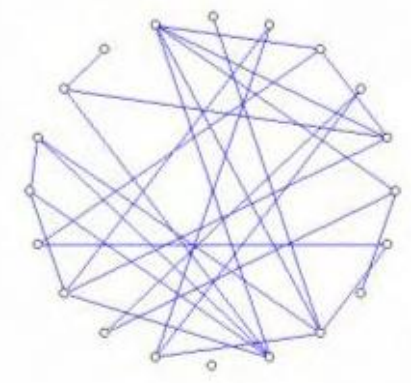

$p=0.2$

(c)

Figure 3. Graphs generated by Erdös and Rényi model ( $p=0, p=0,1, p=0,2)$ 


\section{Results and proofs}

We investigation the Erdős-Rényi model but we suppose that the graph is connected and the graph have dominating set (a dominating set for a graph $G=(V ; E)$ is a subset $D$ of $V$ such that every vertex not in $\mathrm{D}$ adjacent to at least one member of $\mathrm{D}$ ).

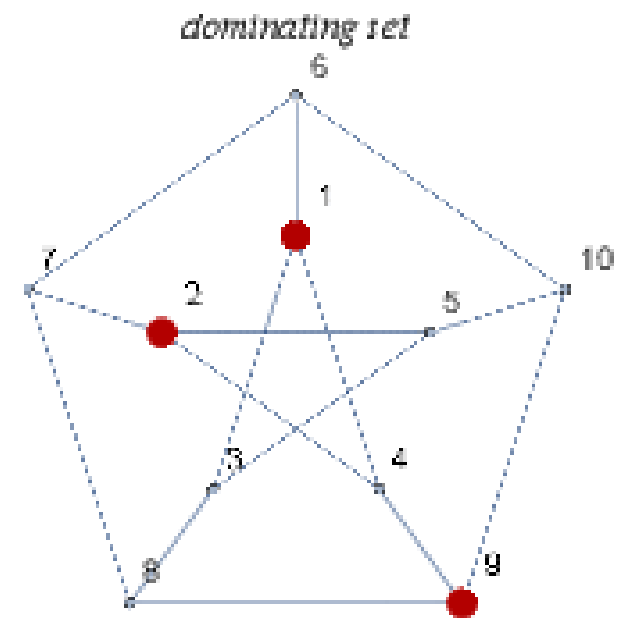

Figure 4. A dominating set in graph (the points of the dominant set are marked in red)

In this model, given two points $\mathrm{x}$ and $\mathrm{z}$, for a third point $\mathrm{y}$, the probability that $\mathrm{y}$ is the common neighbour of them, is $\mathrm{p} 2$, because of independence. If $\mathrm{y}$ runs through all the points different from $\mathrm{x}$ and $\mathrm{z}$, the probability that none of them is a common neighbour of the two fixed points is $\left(1-p^{2}\right)^{n-2}$, from the independence again. Consequently,

Theorem 1. For all $\mathrm{x} \neq \mathrm{z}$, the probability that the distance of $\mathrm{x}$ and $\mathrm{z}$ is at most 2 , is at least $\mathrm{P}(\mathrm{n}):=$ $1-\left(1-p^{2}\right)^{n-2}$. ( $\mathrm{P}(\mathrm{n})$ tends to 1 , of course).

Theorem 2. 'At least' is written here because $\mathrm{x}$ and $\mathrm{z}$ may be adjacent.

First, ordinary domination is considered.

As we know, the probability that the set $\mathrm{D}:=\{1,2, \ldots, \mathrm{r}\}$ is dominating in $\mathrm{G}$ tends to 1 if $\mathrm{r}=\log \mathrm{n}+$ an where $q=p=1 / p$ and an tends to infinity.

Now we add auxiliary edges such that they yield a ( $\mathrm{n}$ arbitrary) tree $\mathrm{T}$ on $\mathrm{D}$. Let us partition the vertex set $\mathrm{V}-\mathrm{D}$ into $\mathrm{r}-1$ (essentially) equal-size classes in such a way that each class $\mathrm{Ci}$ corresponds to some auxiliary edge ei of $\mathrm{T}$ between $\mathrm{xi}$ and $\mathrm{zi}(\mathrm{i}=1,2, \ldots, \mathrm{r}-1)$. The size of one class is about ( $\mathrm{n}-$ $\mathrm{r}) /(\mathrm{r}-1)$, which is asymptotic to $\mathrm{n} / \mathrm{r}$. By Theorem 1, if we pick a pair (xizi), in the subgraph induced by $\mathrm{Mi}:=\mathrm{Ci} \cup\{\mathrm{xi}, \mathrm{zi}\}$ the probability of the event Ai that their distance is at most 2, is at least si := $1-\left(1-p^{2}\right)^{n_{i}}$ where ni :=|Ci|.

If for every $\mathrm{i}$, the event Ai occurs, we may construct a (generally larger) dominating set of G, namely, we add a common neighbour yi of xi and zi in the subgraph G|Mi (or they are adjacent).

Note that, because of the edge-disjointness of the subgraphs induced by the the vertex sets Mi, the events Ai are independent. Consequently,

Theorem 3. The probability that, in such a way, we obtain a connected dominating subgraph, is at least $\prod_{i=1}^{r-1} s_{i}$. 


\section{References}

[1] Bacsó, G., Túri, J., Tuza, Zs. (2021). Connected domination in random graphs, submitted for publication.

[2] Bollobás, B. (2001). Random Graphs. Cambridge University Press. https://doi.org/10.1017/CBO9780511814068

[3] Bonato, A., Wang, C. (2008). A note on domination parameters in random graphs. Discussiones Mathematicae Graph Theory, 28, 335-343. https://doi.org/10.7151/dmgt.1409

[4] Das, B., Bharghavan, V.: Routing in ad-hoc networks using minimum connected dominating sets. 1997 International Conference on Communications, Proceedings of ICC'97, Montreal, Canada, pp. 376-380 Vol.1. 10.1109/ICC.1997.605303

[5] Duchet, P., Meyniel, H. (1982). On Hadwiger's number and stability numbers. Annals of Discrete Mathematics, 13, 71-74. https://doi.org/10.1016/S0304-0208(08)73549-7

[6] Duckworth, W., Mans, B. (2009). Connected domination of regular graphs. Discrete Mathematics, 309, 2305-2322. https://doi.org/10.1016/j.disc.2008.05.029

[7] Feller, W. (1957). An Introduction to Probability Theory and its Applications. Vol. I., Second Edition, Wiley, New York.

[8] Gilbert, E. N. (1959). Random graphs. Annals of Mathematical Statistics, 30, 1141-1144. https://doi.org/10.1214/aoms/1177706098

[9] Haynes, T. W., Hedetniemi, S. T., Slater, P. J. (1998 a). Fundamentals of Domination in Graphs. Marcel Dekker, New York.

[10] Haynes, T. W., Hedetniemi, S. T., Slater, P. J. (eds.) (1998 b). Domination in Graphs: Advanced Topics, Marcel Dekker, New York.

[11] Li, H., Wu, B., Yang, W. (2018). Making a dominating set of a graph connected. Discussiones Mathematicae Graph Theory, 38, 947-962. https://doi.org/10.7151/dmgt.2053

[12] Liu, Z., Wang, B., Guo, L. (2010). A survey on connected dominating set construction algorithm for wireless sensor networks. Information Technology Journal, 9, 1081-1092. https://doi.org/10.3923/itj.2010.1081.1092

[13] Ore, O. (1962). Theory of Graphs, Colloquium Publications. American Mathematical Society, 38, Providence, RI https://doi.org/10.1090/coll/038

[14] Watts, D.J., Strogatz, S. H. (1998). Collective dynamics of 'small-world' networks. Nature, 393 (6684) 440-442. 10.1038/30918. https://doi.org/10.1038/30918

[15] Wieland, B., Godbole, A. P. (2001). On the domination number of a random graph. Electronic Journal of Combinatorics, 8. https://doi.org/10.37236/1581 